https://doi.org/10.48009/4_iis_2019_56-64

Issues in Information Systems

Volume 20, Issue 4, pp. 56-64, 2019

\title{
ARE DRIVERLESS CARS TRULY A REALITY? A TECHNICAL, SOCIAL AND ETHICAL ANALYSIS
}

\author{
Choton Basu, University of Wisconsin-Whitewater, basuc@uww.edu \\ Manu Madan, University of Wisconsin-Whitewater, madanm@uww.edu \\ Balaji Sankaranarayanan, University of Wisconsin-Whitewater, sankarab@uww.edu
}

\begin{abstract}
Autonomous vehicles are increasingly becoming a reality in the near future. Over the years the automobile industry has continued to provide all types of incremental automations to their cars. Recent trends seem to imply that most consumers have accepted that self-driving cars are the future. Previous studies have focused on usage, technical features, policy issues and other dimensions that seem to prioritize the supply side of the industry. Few studies have also started to examine the ethical aspects of this particular technology. Our research study provides a preliminary understanding of the technical, social and ethical barriers that can influence adoption decision in consumers. Our focus was to understand the technical, social and ethical dimensions in a single study. We collected data from 282 individuals and were able to demonstrate that technical, social and ethical barriers will play a significant role in the adoption of the autonomous vehicles. We also found that a majority of the people would like to be involved in the decision making if the car had to react to a critical situation. In other words, most people were not yet prepared to let the car make the decision on who survives but if it does, it needs to minimize the harm.
\end{abstract}

Keywords: Driverless, Autonomous Vehicles, Social, Adoption, IT, Behavioral Intent and Ethics

\section{INTRODUCTION}

A driverless car (DC) is an unmanned vehicle that is capable of maneuvering without human input but utilizes the support of several sophisticated sub-systems and devices (Owczarzak \& Żak, 2015). Autonomous car technology has been in development since 2009 and in 2013, the US Department of Transportation's National Highway Traffic Safety Administration (NHTSA) defined various levels of autonomous driving, officially adopting it into their policy in 2016 (Reese,2016). Level 0 is a fully driver-operated vehicle, levels 1 through 3 are semi-autonomous, and levels 4 and 5 are fully autonomous vehicles, with level 5 meaning the vehicle could do anything a human driver could do in any scenario. Many automotive OEMs are optimistic about Level 5 releases by 2021.

Over the years the current automobile industry has continued to provide all types of incremental automations to their cars. It started with simple automation of remote starts, back-gate sensors, and various maintenance warnings. Recent trends include collision avoidance system, adaptive cruise control, heads-up display, and lane change assistance are all examples of driver assistance systems that are currently available commercially (Zindler \& Geiss, 2016). Over the years these features have enabled the development of the fully autonomous vehicle (AV).

Information systems literature has dealt extensively with the factors that contribute to the adoption of a particular technology. Prior research has provided individual, group and organizational-level factors that contribute to technology adoption in a mandatory, as well as voluntary environment. Technology Acceptance Model (TAM), based on the theory of planned behavior (Mathieson, 1991), posits that two factors: ease of use and usefulness of a technology, and their antecedents, can predict the behavioral intent to adopt a particular technology (Davis et al. 1992; Venkatesh and Davis, 2003). Later work by Venkatesh and colleagues on UTAUT and UTAUT2 extends this framework to include various individual and social factors such as effort expectancy, performance expectancy and social influence as antecedent factors that predict the behavioral intent to adopt technology in a consumer context (Venkatesh et al. 2003; Venkatesh et al. 2012).

But what happens when the adoption of technology involves possible danger to self and others? Now we are faced with the situation where technology adoption models need to consider other dimensions. This research shows that 


\section{Issues in Information Systems \\ Volume 20, Issue 4, pp. 56-64, 2019}

technological, social and ethical factors can play a significant role in influencing an individual to adopt a technology in this context. While previous research has looked at applications, usage, technical features, ease of use, usefulness, we were particularly interested to understand if people would even be willing to get in an autonomous vehicle. Also, how they would respond if the risks were increased for example by having your family use the automated car. To us this presented some exciting research gaps that could focus on technical, social and ethical issues in a single study.

\section{An Ethical Dilemma}

Recent research has expanded prior work on IT adoption and post-adoptive use to include ethical concerns of individuals. In a study on autonomous vehicles and their use, Bonnefon et al. (2016) tested this ethical dilemma using a simulation-based field experiment. Two types of situations were modeled: one in which the maximum number of people were saved (utilitarian), and the other where the passengers in the vehicle were saved (self-protective). Participants stated that the utilitarian vehicle was more moral and would recommend to others. However, they still chose the self-protective vehicle, if they themselves were the intended passengers of the vehicle. That is, the utilitarian vehicle was rated as highly moral only if it applied to others. This presents a stark ethical dilemma in the minds of customers in terms of how autonomous vehicles will and should behave in such situations.

Autonomous vehicles are dependent on artificial intelligence and machine learning technology (Shariff et al. 2017), which can evoke significant technology-related, social and ethical issues in the minds of customers. Artificial intelligence and machine learning refer to a variety of statistical tools and techniques that accept inputs and provide processing capabilities to an autonomous vehicle. Inputs obtained from sonar, cameras, or laser technologies are processed by the artificial intelligence engine to help the vehicle to avoid collisions, prevent drifting from lane, detect blind spots etc. (Davies, 2018). Some of these features are already available in cars today with the exception that the decision-making regarding driving is left to the individual. However, in autonomous vehicles, the decision-making is also handled by the AI and machine-learning engine. There are many things that the current technology has yet to discover on how to handle driving with driverless vehicles. One of the big issues is that the further we move into automation, the less attention a human will pay. The different levels of automations out there require different levels of attention from the driver to maintain safety of the vehicle.

There are three clear ways to compartmentalize the problem - technical dimension, social barriers and the ethical considerations of using the technology. The legal or regulatory dimension was not considered in this study since we were focusing primarily on the consumer perspective. We also did not consider the actual use of the autonomous vehicle (AV).

Technical dimension focuses on the level of confidence the consumer has with the technology. The ability of the technology to respond to changes in the environment, surroundings, conditions and adapt to them. Technology in these situations are designed by engineers who rely on deterministic processes to build solutions. However, for driverless cars to work one has to take into account the random nature of human behavior this leading to probabilistic models that shape the performance of the car. Therefore, technical topics come to the forefront when incidence of death resulting from self-driving cars emerge as headlines in the news. These cars are dependent on sensors, cameras, \& radar to gather data. The vehicle can make an incorrect decision like in the example with Uber in Tempe, AZ where the vehicle did not determine the object in front of it as a pedestrian. The incident report concluded "a preliminary investigation showed that the vehicle was moving around 40 miles per hour when it struck Ms. Herzberg, who was walking with her bicycle on the street. He said it did not appear as though the car had slowed down before impact and that the Uber safety driver had shown no signs of impairment. The weather was clear and dry" (Wakabayashi, 2018). This has a direct impact on the comfort level of the consumers to start using these vehicles. The question becomes is the technology ready or is there need for further testing? It is clear that most consumers see this technology still in early stages, but the supply side of the technology such as engineers, manufacturers and other advocates are convinced that the technology is ready.

The social dimension has massive implications when we consider the operation the current versions of self-driving vehicles. It is estimated that over 37,000 Americans and 1.3 million people globally die every year in automobile accidents. This drives the case for autonomous vehicles because while human driving error is not the sole cause of all 1.3 million deaths, some estimates put the number of accidents committed through human error as high as $94 \%$. Selfdriving cars might not be able to eliminate all driving fatalities, but couldn't they lower the number? Another argument for the benefits is that these vehicles can prevent situations where a driver's driving ability is impaired or a driver is 


\section{Issues in Information Systems}

Volume 20, Issue 4, pp. 56-64, 2019

not paying enough attention. Moreover, as the technology becomes more advanced, it becomes more of a system increasingly monitored by human (Shariff, A. et. al., 2017). In other words, there are massive social implications of this technology because it not only impacts the driver, the passengers but also people around them. This research focused on the driver and their immediate family as the social context.

The final dimension to consider are the ethical components of adoption of such technology. Shariff, A. et. al, (2017) have been conducting a series of research projects related to ethical aspects of autonomous vehicles and the policies as well as regulations that need to be in place for such technology. With new technology, it is unclear how much government regulations are adequate. Some regulations are already in place with laws of the road, but most people realize that additional regulations are needed. However, in countries such as the USA "too-much" government regulations are frowned upon but everything changes if the new technology is viewed as a risk to self and others. The NCLS detailed the legislation and orders signed by 33 states as well as at the federal level. These range from creating safety committees to defining the pilot phase of the innovation. There are many new questions to resolve: Should there be regulations on software updates for the vehicles to be on the road? How often should a vehicle's AI system be tested? What regulations should be in place to account for weather conditions? One of the biggest issues - who should be held liable in the instance of a fatality? Manufacturer, the AI design company, the owner of the vehicle and so on. Of course, this also has massive implications of the car insurance industry.

Therefore, prior research shows that barriers to adoption in terms of technology, social and ethical concerns can significantly affect an individual's decision to adopt an autonomous vehicle. In the next section, we develop and test a research model based on these perspectives.

\section{RESEARCH MODEL}

Figure 1 shows the research model for this study. Behavioral Intent (BI) to adopt is considered as the dependent variable (DV) in this study. The model shows that ethical barriers, technology trust barriers and social barriers are negatively related to behavioral intent to adopt. We discuss the hypotheses development below.

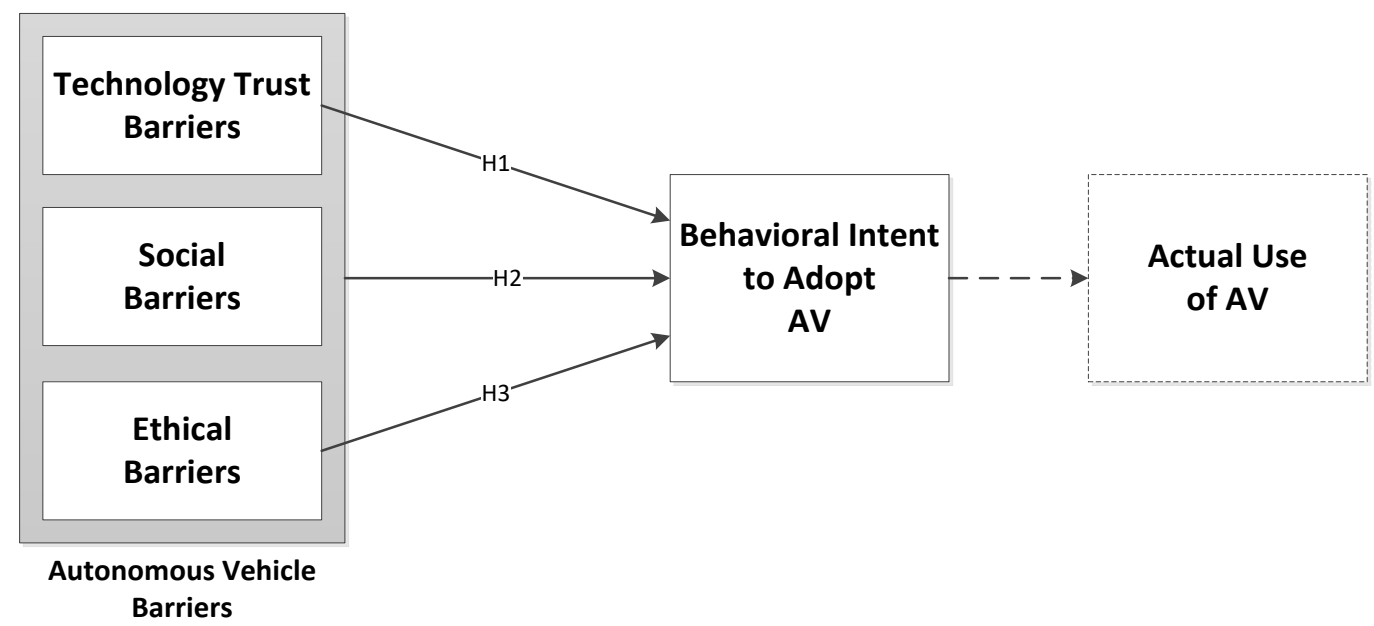

Figure 1. Research Model on Intention to Adopt AVs (*Actual use not considered in this study) 


\section{Impact of Technology Trust Barriers}

Technology trust barriers refer to the perceived trust placed in the AI software and associated hardware factors by an individual, for the potential use of autonomous vehicles (AVs). Trust in technology has been considered by prior research on IT adoption as one of the important antecedents of the intention to adopt an IT system (Bahmanziari et. al. 2003). Recent research on driverless cars also discusses the role of technology factors related to privacy and security as barriers to adoption (Kaur and Ramprasad, 2018). In the case of AVs, the use of AI has led to questions on the trustworthiness of the technology. Practitioner research on AI also lends credence to this idea of a general lack of adoption due to fundamental barriers to adoption (McKinsey 2018).

We posit that technology trust barriers can negatively impact the intent to adopt an autonomous vehicle. The foundations of technology in an autonomous vehicle are artificial intelligence (AI) and machine learning which are maturing at a rapid pace. However, industry experts and practitioners contend that AI used in these vehicles has not reached its full predictable use. Recent incidents of accidents with autonomous vehicles (Wakabayashi, 2018) raise questions on the safety aspects of the technology and tendency to commit mistakes. This suggests that AVs have to resolve technological issues before they can be rolled out to the general public. Shariff et al. (2017) state that the lack of transparency in decision-making processes could inhibit an individual from completely trusting the autonomous vehicles. Therefore, we posit that:

\section{H1: Technology trust barriers will have a negative impact on customers' intent to adopt an autonomous vehicle.}

\section{Impact of Social Barriers}

Social barriers refer to the perceived inhibitions due to existing cultural norms and values, associated with the potential use of AVs. Technology adoption theories such as UTAUT (Venkatesh et al. 2003) have documented the positive impact of social influence on adoption decisions in individuals. Cultural norms and values can be influenced by the environment and can subsequently influence behaviors related to technology (Srite and Karahana, 2006). For example, in some countries, cell phone use in universities and schools are prohibited, since culturally, cell phones are considered to be distracting to the purpose of education.

We posit that social barriers will have a negative impact on the adoption of AVs. In his seminal work on innovation constraints, Owens (2012) discusses the societal constraints that hamper the adoption and diffusion of an innovative technology or product. For instance, when Sow-N-Gro - a product that had elicited positive response - was revealed to contain human hair, the customer's enthusiasm vanished. In other words, as the cultural norms and values about the use of a human hair were brought into question, the product garnered a negative reaction. We subscribe to the view theorized by Owens (2012), and posit that social barriers can have a significant negative influence on adoption of AVs.

\section{H2: Social barriers will have a negative impact on the customers' intent to adopt an autonomous vehicle.}

\section{Impact of Ethical Barriers}

Ethical barriers refer to the perceived ethical and moral inhibitions associated with the potential use of autonomous vehicles. Information systems ethics has studied the ethical aspects of systems use from an individual and organizational perspective. This stream of literature has studied the impact of ethical rules and rule-making, types of ethics and systems, prevention, detection and correction of fraudulent transactions, and individual user resistance (Chouduri and Zamani, 2016, Hirschheim and Klein 1994, Miranda et. al. 2016). As noted earlier, the ethics of using autonomous vehicles is explored extensively in a study by Bonnefon et al. (2016) where they delve into a scenariobased experiment modeling different ethical dilemmas. This study shows that customer preferences to adopt can be deeply rooted in ethical concerns about AV use. 
We posit that ethical barriers can have a negative impact on the individual adoption of autonomous vehicles. Ethical concerns regarding autonomous vehicles are deeply rooted in the attitudes of individuals (Bonnefon et al. 2016), and have a strong influence on the behaviors of an individual. For instance, individuals are found to resist the adoption of a new IT system, when the system acts to control their behavior (hegemony), as against enabling it (emancipatory) (Miranda et al. 2016). Similarly, when an emerging technology evokes ethical and moral inhibitions, an individual might be hesitant to adopt such technology. Therefore, we posit that:

\section{H3: Ethical barriers will have a negative impact on customers' intent to adopt an autonomous vehicle.}

In the following sections, we present details of our research methodology, data collection, analysis and results. We also present a comprehensive discussion on our findings and exciting future research on this topic in the summary section.

\section{RESEARCH METHODOLOGY}

The goal of this project was to stay focused on the technical, social and ethical dimensions but also keep it simple to enable respondents to respond to our questions. Data was collected over a semester in a combined technology course at a mid-sized university in the United States. The demographics of the respondents are provided in Table 1 (below).

Table 1. Respondent Demographics

\begin{tabular}{|l|l|c|}
\hline Gender & Undergraduate Program & Responses \\
\hline Female & Business Major & 82 \\
\hline & $\begin{array}{l}\text { Non-business major or } \\
\text { Undecided }\end{array}$ & 8 \\
\hline Male & Business Major & 177 \\
\hline & $\begin{array}{l}\text { Non-business major or } \\
\text { Undecided }\end{array}$ & 15 \\
\hline $\begin{array}{l}\text { Majority of the respondents were between the ages of } \\
\text { 19-24 with few non-traditional students. Data was } \\
\text { collected in a required Information Technology course } \\
\text { at the end of the semester. Total responses = 282 }\end{array}$ \\
\hline
\end{tabular}

As a first step we asked all participants in the study to watch a TED talk video by Iyad Rahwan titled: What moral decisions should a driverless car make? The link was provided to the respondents and they were given one week to watch the video and then respond to five questions posed by the researchers.

Link: https://www.ted.com/talks/iyad_rahwan_what_moral_decisions_should_driverless_cars_make?

The five questions posed for the survey were as follows:

1. Would you buy a driverless car? This was our dependent variable represented as the Adoption of the Autonomous Vehicle in our research model. (Dependent Variable for Model 1)

2. Do you think others should be buying driverless cars? Adoption of AV by others (Dependent Variable for Model 2)

3. Are you willing to be a passenger in a driverless car? This was our question to test the underlying confidence the respondent/consumer had with the Technology of the AV. We noted that a significant number of respondents commented that they were not comfortable with the current technology and that it needed more testing. 
4. Would you be comfortable with letting your family members ride in a driverless car? This was our question on the Social Barrier for adoption since it forced the respondent face the issue of safety with respect to their family. The goal here was to have them respond to a situation where the adoption would be for someone else, in this case their family.

5. How would you approach the ethical issue posed by the presenter? Think carefully and answer. The question posed by the presenter was an ethical and moral dilemma - Should a driverless car kill you (the passenger) if it means saving five pedestrians? The students were asked to respond to this question. This question was our qualitative examination of the ethical barrier. Harari (2018), presents a compelling argument for engineers who design these driverless cars to have a deep understanding of human nature and ethical behavior. It is not just a technical solution. He does not particularly address the consumer side of the equation. Therefore, we wanted to give the respondents the ability to see other responses and comment on them after they had completed responding to the five questions.

We measured the behavioral intent to adopt an autonomous vehicle using the first two questions. First, we measured the individual's intent to purchase the vehicle with the question: "Would you buy a driverless car?" We also captured the behavioral intent of the individual to recommend to others by asking if others should be buying driverless cars.

Technology trust barriers were captured by asking if the individual will be willing to be a passenger in a driverless car. The assumption is that if the individuals trust technology, they would be willing to be a passenger in the car. Social barriers sought to capture cultural norms by asking if they would be comfortable with letting their family members ride in a driverless car. Ethical barriers were captured with the open-ended question "How would you approach the ethical issue posed by the presenter?" As we noted earlier the question was based on the video on whether the driverless car should make the decision during a possible car crash to decide - whether to swerve to avoid the accident but killing the passengers in the process or taking action to protect the passengers and possibly killing pedestrians or others outside the car. As indicated in Table 1, we received 282 completed online surveys that were usable. The results below are based on our analysis of this data.

\section{RESULTS}

We coded technology trust barriers, social barriers and the dependent variables as a $0-1$ variable ( 0 for No, 1 for Yes). We conducted a linear regression analysis of the impacts of technology trust barriers and social barriers on the behavioral intent to adopt an autonomous vehicle. The two questions for behavioral intent were treated as two separate dependent variables. Table 2 shows the results of regression analysis for each of these two dependent variables. Results show that technology trust barriers have a significant negative impact on the behavioral intent to purchase a vehicle $(\beta=-0.289, \mathrm{p}<0.001)$. Further, technology trust barriers were also found to have a significant negative impact on behavioral intent to recommend a vehicle $(\beta=-0.249, \mathrm{p}<0.001)$. Therefore, hypothesis H1 is supported. Similarly, social barriers were found to have a negative impact on the behavioral intent to purchase a vehicle $(\beta=-0.419, \mathrm{p}<0.001)$ and behavioral intent to recommend a vehicle $(\beta=-0.317, \mathrm{p}<0.001)$. Therefore, hypothesis $\mathrm{H} 2$ is also supported.

Table 2. Regression Analysis

\begin{tabular}{lcc}
\hline & Model 1 & Model 2 \\
\cline { 2 - 3 } & DV: BI to Purchase & DV: BI to Recommend \\
\hline Intercept & $\mathbf{0 . 7 4 4} * * *$ & $\mathbf{0 . 9 4 7 * * *}$ \\
Technology Trust Barriers & $\mathbf{- 0 . 2 8 9 * * *}$ & $\mathbf{- 0 . 2 4 9} * * *$ \\
Social Barriers & $\mathbf{- 0 . 4 1 9 * * *}$ & $\mathbf{- 0 . 3 1 7 * * *}$ \\
\hline
\end{tabular}

Note: BI is Behavioral Intent, DV is Dependent Variable

We also spent a significant amount of time analyzing responses to question \#5. Some of the key responses from that are provided below. Our interpretation and analysis of question $\# 5$ follows. 
Harari (2018) said, "We are now facing not just a technological crisis but a philosophical crisis." The question of ethics has become a dominant area of research for autonomous vehicles. By enabling our respondents to share their decision to the question posed by the presenter, we not only were able to gather their decision but also in the majority of the cases, their thought-process. Below, we have highlighted some of the more brilliant responses:

- I would want the car to choose the approach that best protects the pedestrian because the passenger in the driverless car knew the risks when he got in the car.

- I would lean to Bentham's Utilitarian approach, as it would be better for humanity as a whole.

- I would want the car to have the safest outcome that there could possibly be. Minimizing the amount of death would be caused by the accident would be the best-case scenario.

- I would not feel comfortable having to be a passenger in a driverless car, reacting one way, and having my car react the other way. I have a problem with AI making life and death decisions that humans are capable of making. It's a decision I couldn't imagine living the rest of my life with if I knew my car hurt or killed someone else that I know I could have averted if I was in control of my car. If driverless cars really are the future and that's where we're moving, I wouldn't know how to program a car on ethics and I pray that other people know how to do so causing the fewest casualties.

There were only a few individuals (less than 12) who wanted to protect themselves as the driver of the car and face whatever consequences if the car hit other people. Their justification was that self-preservation is the key and the vehicle needs to be designed to protect the driver or passenger first.

Overall it is clear that this is serious ethical dilemma for most consumers. Most consumers want to do reduce the amount of damage or harm from the technology. Several responses also tried to differentiate between adults and children getting hurt. We find the social aspects start to work its way into the ethical dilemma. But clearly the results indicate that minimizing the amount of death or harm should be the goal of the technology in this case an autonomous vehicle.

\section{SUMMARY}

The purpose of this research study is highlight the three basic barriers - technical, social and ethical barriers - that have a significant impact on the adoption of autonomous vehicles. This study therefore makes key contributions to extant academic and practitioner literature.

Our study reveals that technology trust barriers have a significant impact on behavioral intent to adopt an autonomous vehicle (AV). This extends prior research on trust and technology, by delving exclusively in the domain of emerging technologies such as AI and machine learning. Future research can delve into the dimensions of trust and technology, and their impacts on adoption. The threat of hacking has been recently demonstrated, where with a few clicks, an autonomous vehicle can be made to speed-up, slow down or stop (Berk, 2019). This suggests that the threat of hacking can be a significant concern in terms of adoption by customers. Therefore, future work can extend our work by focusing on aspects of privacy and security as applied to autonomous vehicles.

This study also shows that social barriers have a significant impact on behavioral intent to adopt an autonomous vehicle (Harari, 2018). This extends prior research on the social influence on IT adoption, by providing a nuanced perspective of the norms and values at play in these settings. Future research can potentially look at the influence of family members and the network to study their impacts on the adoption decision. As evidenced in prior studies and the work on innovation (Owens 2012), societal constraints need to be resolved before an innovation can be successful. Further, findings from our study show that ethical barriers have a significant impact on behavioral intent to adopt an autonomous vehicle. Ethical issues in information systems have predominantly been focused on post-adoptive use situations. Our study contributes to this stream of literature by focusing on the adoption decision as related to ethical considerations of technology. We believe that ethical concerns regarding autonomous vehicles will be notably important in the future. Therefore, future research can delve into more ethical concerns with technology, adoption and use of autonomous vehicles. 
In summary, our research study provides a preliminary understanding of the technical, social and ethical barriers that can influence adoption decision in consumers. Autonomous vehicles are increasingly becoming a reality in the near future. As car manufacturers and technology companies strive to introduce autonomous vehicles, we believe that our study and this stream of research can enable them to address the customer concerns effectively.

\section{REFERENCES}

Bahmanziari, T., Pearson, M. J. \& Crosby, L. (2003). Is Trust Important in Technology Adoption? A Policy Capturing Approach, Journal of Computer Information Systems, 43(4), 46-54.

Berk, B. (2019). We Hack a Car (It's Way Easier than You Might Think), Accessed from: https://www.automobilemag.com/news/car-hacking-we-hack-autonomous-car/, Last Accessed on July 10, 2019.

Bonnefon, J-F., Shariff, A., \& Rahwan, I. (2016). The Social Dilemma of Autonomous Vehicles. Science, 352(6293), 1573-1576.

Choudrie, J., \& Zamani, E. D. (2016). Understanding Individual User Resistance and Workarounds of Enterprise Social Networks: The Case of Service Ltd., 31(2), 130-151.

Davies, A. (2018). The Wired Guide to Self-Driving Cars, Accessed at: https://www.wired.com/story/guide-selfdriving-cars/, Last accessed on July 10, 2019.

Harari, Y. N. (2018). 21 lessons for the 21st century. First edition. New York: Spiegel \& Grau.

Hirschheim, R., \& Klein, H.K. (1994) Realizing emancipatory principles in information systems development: the case for ETHICS. MIS Quarterly, 18(1), 83-109.

Kaur, K., \& Ramprasad, G. (2018) Trust in driverless cars: Investigating key factors influencing the adoption of driverless cars. Journal of Engineering and Technology Management, 48(2), 87-96.

Konig, M. \& Neumayr, L. (2017). Users' resistance towards radical innovations: The case of the self-driving car. Transportation Research Part F, 44, 42-52.

Koul, S. \& Eydgahi, A. (2018). Utilizing Technology Acceptance Model (TAM) for driverless car technology adoption. Journal of Technology Management and Innovation, 13(4), 37-46.

Mathieson, K. (1991). Predicting user intentions: comparing the technology acceptance model with the theory of planned behavior. Information Systems Research, 2(3), 173-191.

McKinsey (2018). Link to access article: https://www.mckinsey.com/featured-insights/artificial-intelligence/aiadoption-advances-but-foundational-barriers-remain, Last accessed: July 10, 2019.

Miranda, S. M., Young, A., \& Yetgin, E. (2016). Are Social Media Emancipatory or Hegemonic? Societal Effects of Mass Media Digitization in the Case of the SOPA Discourse. MIS Quarterly, 40(2), 303-329. 
Owczarzak, Ł. and Żak, J. (2015). Design of passenger public transportation solutions based on autonomous vehicles and their multiple criteria comparison with traditional forms of passenger transportation. Transportation Research Procedia, 10(2015), 472-482. https://doi.org/10.1016/j.trpro.2015.09.001

Owens, D. A. (2012). Creative People Must be Stopped: 6 Ways We Kill Innovation, San Francisco, CA: JosseyBass.

Panagiotopoulos, I., Dimitrakopoulos, G. (2018). An empirical investigation on consumers' intentions toward autonomous driving. Transportation Research Part C 95, 773-784.

Reese, H. (2016, January 20). Updated: Autonomous driving levels 0 to 5: Understanding the differences. Retrieved October 11, 2018, from http://www.techrepublic.com/article/autonomous-driving-levels-0-to-5understanding-the-differences/

Shariff, A., Bonnefon, J- F., \& Rahwan, I. (2017) .Psychological roadblocks to the adoption of self-driving vehicles. Nature Human Behavior, 1(3), 694-696.

Srite, M., and Karahana, E. (2006) The Role of Espoused National cultural values in Technology Acceptance. MIS Quarterly, 30(3), 679-704.

Venkatesh, V., Morris, M.G., Davis, G.B., \& Davis, F.D. (2003). User Acceptance of Information Technology: Toward a Unified View. MIS Quarterly, 27(3), 425-478.

Venkatesh, V., Thong, J. Y. L. \& Xu, X. (2012). Consumer acceptance and use of information technology: extending the unified theory of acceptance and use of technology. MIS Quarterly, 36(1), 157-178.

Wakabayashi, D. (2018). Self-Driving Uber Car Kills Pedestrian in Arizona, Where Robots Roam, Accessed from https://www.nytimes.com/2018/03/19/technology/uber-driverless-fatality.html, Last accessed July 10, 2019.

Zindler, K. \& Geiss, N. (2016). Vehicle ego-localization in autonomous lane-keeping evasive maneuvers. IFACPapersOnLine, 49(11), 160-167. https://doi.org/10.1016/j.ifacol.2016.08.025 